\title{
EXTRACCIÓN ASISTIDA POR ULTRASONIDO DE COMPUESTOS FENÓLICOS DE LA CÁSCARA DE SANKY (Corryocactus brevistylus)
}

\author{
Tatiana Rojas ${ }^{\mathrm{a}}$, María E. Fuentes Campos ${ }^{\mathrm{b} *}$, Eliana Contreras-López ${ }^{\mathrm{c}}$,

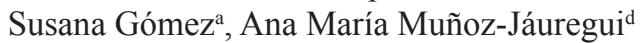

\begin{abstract}
RESUMEN
La extracción por ultrasonido permite recuperar sustancias bioactivas de los residuos agroindustriales, además es una técnica rápida, limpia y amigable con el medio ambiente. Se realizó la extracción de componentes fenólicos por ultrasonido a las cáscaras del fruto del sanky (Corryocactus brevistylus). Esta planta es una cactácea que crece en los Andes del Perú y su fruto es consumido por las comunidades. La cáscara de sanky reportó: humedad 10,74 \%; proteína $9,19 \%$; cenizas $14,75 \%$; grasa $2,68 \%$; fibra $16,39 \%$ y extracto libre de nitrógeno 46,25 \%. En las semillas, se encontró 207,81 ppm de calcio, 39,36 ppm de hierro y 9,4 ppm de zinc. Para la extracción se evaluaron tres factores y cada uno en tres niveles: concentración del solvente etanol (40, 50 y $60 \%)$, tiempo (20, 30 y 40 minutos) y temperatura $(25,35$ y $\left.45^{\circ} \mathrm{C}\right)$. Se empleó el diseño de Box Behnken, reportando 15 ensayos experimentales. Se evaluó los polifenoles totales por el método de Folin Ciocalteau. El tratamiento M11 (50\% $\mathrm{v} / \mathrm{v}$ de etanol, 40 minutos y $25^{\circ} \mathrm{C}$ ) reportó $43,9 \mathrm{mg}$ ácido gálico/g muestra seca y fue el más eficiente. Los siguientes tres tratamientos M3 $\left(60 \% \mathrm{v} / \mathrm{v}, 40\right.$ minutos, $\left.35^{\circ} \mathrm{C}\right)$; M6 $(40 \% \mathrm{v} / \mathrm{v}, 30$ minutos, $\left.45{ }^{\circ} \mathrm{C}\right)$ y M9 $\left(50 \% \mathrm{v} / \mathrm{v}, 20\right.$ minutos, $\left.25^{\circ} \mathrm{C}\right)$ reportaron valores de 31,$5 ; 32,9$ y 32,6 $\mathrm{mg}$ ácido gálico/g muestra seca, respectivamente, y no tuvieron diferencias significativas. La variable más importante fue el tiempo de extracción, luego la concentración del solvente. El objetivo fue determinar las variables de concentración de solvente, tiempo y temperatura en la extracción asistida por ultrasonido para recuperar los componentes fenólicos de la cáscara de sanky.
\end{abstract}

Palabras claves: ultrasonido, compuestos fenólicos, sanky, sustancias bioactivas.

\footnotetext{
a Departamento de Química, Facultad de Ciencias, Universidad Nacional Agraria La Molina. Av. La Molina s/n, La Molina. Lima, Perú.

b*Facultad de Química e Ing. Química, Universidad Nacional Mayor de San Marcos. Av. Universitaria /Calle Germán Amézaga 375. Edificio Jorge Basadre Ciudad Universitaria, Lima, Perú, elifuentesc@gmail.com.

c E.P. Ciencia de los Alimentos. Facultad de Farmacia y Bioquímica, Universidad Nacional Mayor de San Marcos, Jr Puno 1002, Lima, Perú.

d Vicerrectorado de Investigación, Universidad San Ignacio de Loyola. Av. La Fontana 550, La Molina. Lima, Perú.
} 


\title{
ULTRASOUND-ASSISTED EXTRACTION OF PHENOLIC COMPOUNDS OF THE PEEL OF SANKY (Corryocactus brevistylus)
}

\begin{abstract}
The ultrasound-assisted extraction allows to recover bioactive substances in the agroindustrial waste, besides, is a fast, clean and friendly with the environment technique. The extraction of total phenolic components by ultrasound was carried out on the peel of the fruit of the sanky (Corryocactus brevistylus). This plant is a cactus that grows in the Andes of Peru and its fruit is used by the communities. The sanky peel reported: humidity $10,74 \%$; protein $9,19 \%$; ashes $14,75 \% ; 2,68 \%$ fat; fiber $16,39 \%$ and nitrogen-free extract $46,25 \%$. The sanky seeds reported 207,81 ppm of calcium, 39,36 ppm of iron and 9,4 ppm of zinc. For the extraction, three factors and each one in three levels were evaluated: solvent concentration (40\%, $50 \%$ and $60 \%)$, time (20, 30 and 40 minutes) and temperature $\left(25,35\right.$ and $\left.45{ }^{\circ} \mathrm{C}\right)$. The design of Box Behnken was used, reporting 15 experimental trials. The total polyphenols was evaluated by the Folin Ciocalteau method. The M11 (50\% v/v ethanol, 40 minutes and $25{ }^{\circ} \mathrm{C}$ ) treatment reported $43,9 \mathrm{mg}$ galic acid/g dry sample, and was the most efficient. The following three treatments: M3 $\left(60 \% \mathrm{v} / \mathrm{v}, 40\right.$ minutes, $\left.35^{\circ} \mathrm{C}\right)$; M6 $(40 \% \mathrm{v} / \mathrm{v}, 30$ minutes, $\left.45^{\circ} \mathrm{C}\right)$ and $\mathrm{M} 9\left(50 \% \mathrm{v} / \mathrm{v}, 20\right.$ minutes, $\left.25^{\circ} \mathrm{C}\right)$ did not show significant differences, reporting values of 31,$5 ; 32,9$ and $32,6 \mathrm{mg}$ galic acid/g dry sample, respectively. The most important variable was the extraction time, then the concentration of solvent. The objective was to determine the variables of solvent concentration, time and temperature during the ultrasoundassisted extraction to recover the phenolic components of the peel of sanky.
\end{abstract}

Key words: ultrasound, sanky, polyphenols, bioactive substances.

\section{INTRODUCCIÓN}

La extracción de componentes bioactivos por ultrasonido es un método limpio, sencillo, rápido y verde en comparación con los métodos convencionales. Además de su alta reproducibilidad en corto tiempo, de fácil manipulación y disminución en el uso de solventes frente a otros métodos ${ }^{1}$. Las ondas de ultrasonido causan la ruptura mecánica de la pared celular liberando los componentes bioactivos, a su vez el calentamiento local del solvente aumenta la difusión del extracto, mejorando así la transferencia de masa a través de la interfase sólido-liquido. Los efectos mecánicos de la sonicación inducen a una mayor disolución del solvente en las paredes y membranas celulares, facilitando la liberación del contenido de las células y mejorando la transferencia de masa ${ }^{2}$.

El sanky (Corryocactus brevistylus) es una cactácea nativa de los Andes peruanos, de tallo carnoso que crece entre los 2500 a $3500 \mathrm{msnm}$, sus frutos son unas bayas de forma redondas, de color verde-amarillo, con espinas. Ayuda en el tratamiento para la prostatitis, problemas 
del riñón y por su aporte de calcio podría ayudar a combatir la osteoporosis. Su fruto presenta propiedades funcionales y terapéuticas, se suele utilizar para la producción de jaleas, néctares, jugos, ensaladas y otros ${ }^{3}$. Este fruto, es conocido, por los lugareños, como el "quitahambre" y suelen consumirlo para largas jornadas de trabajo. Sin embargo, la cáscara del fruto es poco valorada, por la falta de información y podría considerarse como una fuente de sustancias bioactivas.

Al respecto, Matos et al. ${ }^{4}$ realizaron la extracción de componentes fenólicos de la pulpa de sanky empleando el método convencional a una temperatura de $70^{\circ} \mathrm{C}$; relación materia prima: solvente de 1:10 (p/v) y etanol al $90 \%$. La capacidad antioxidante de estos componentes fenólicos por el método de DPPH reportó 439,11 ugTrolox/g muestra. Zou et al..$^{5}$ optimizaron la extracción asistida por ultrasonido de los fenoles y flavonoides de hojas de mango (Mangifera indica L.), las variables evaluadas fueron temperatura, tiempo de extracción y concentración de alcohol en el solvente. La temperatura y el tiempo de extracción fueron los dos factores más importantes.

Existen pocas investigaciones sobre la cáscara de sanky, considerando que podría utilizarse como ingrediente y/o insumo en el procesamiento de alimentos. Al respecto, Lipe ${ }^{6}$ reportó la presencia de un hidrocoloide en el zumo de sanky que tendría un efecto protector para el hígado y la mucosa gástrica.

Dentro de los residuos agroindustriales, el grupo de alimentos con mayor porcentaje de pérdidas y desperdicios es el de frutas y verduras (55\%), seguido de raíces y tubérculos (40 \%). Los residuos provenientes del procesamiento de frutas, son poco valorados al desconocer su composición, cantidad y calidad de sus componentes ${ }^{7}$. Los polifenoles son compuestos que se encuentran en diferentes partes de las plantas y también en los residuos, presentan un efecto protector contra los radicales libres, además retardan el avance de algunas enfermedades crónicas. Estos compuestos han demostrado una intensa actividad biológica, como antioxidantes, antibacterianos e incluso antitumorales ${ }^{8}$. En las cáscaras de varios frutos, se reporta una mayor concentración de sustancias bioactivas que en la misma pulpa de la fruta, en el caso de las cáscaras de plátano, sandía y uva reportaron 24,42; 26,73 y 113,40 mg ácido gálico/g de muestra, respectivamente ${ }^{7}$; así como la cáscara de mandarina común y de toronja que, según Ordóñez-Gómez et al. ${ }^{9}$, presentaron 3,22 y 3,08 mg ácido gálico/g muestra, respectivamente. Respecto a la cáscara del fruto del sanky, a la fecha no hay información sobre su composición y/o aplicaciones en algún tipo de industria.

Al eliminar estos residuos también se estaría eliminando una posible fuente de sustancias biológicamente activas que podrían generar un valor agregado a estos residuos y podrían ser utilizados como ingredientes en la industria de alimentos, farmacéutica, cosméticos y también nutraceútica. Según el reporte de Waste Atlas $2013^{10}$, en el Perú se producen 54,8 \% de desechos orgánicos. Asimismo, se estaría tratando los residuos sólidos orgánicos de una manera sostenible y amigable con el cuidado del medio ambiente. De allí la importancia de evaluar los componentes fenólicos presentes en la cáscara de sanky aplicando una extracción asistida por ultrasonido. 
El objetivo de la investigación fue aplicar la extracción asistida por ultrasonido para recuperar los componentes fenólicos de la cáscara de sanky utilizando como variables la concentración de solvente, tiempo y temperatura de extracción.

\section{PARTE EXPERIMENTAL}

\section{Materia prima y reactivos}

Se eliminaron aquellos frutos de sanky con daños físicos, luego se lavaron con agua destilada, se secaron con papel toalla y se pelaron manualmente. Las cáscaras fueron secadas en estufa a $35^{\circ} \mathrm{C}$, luego fueron molidas y almacenadas en empaques herméticos y asépticos. El etanol (99\%), ácido sulfúrico (99\%), hidróxido de sodio y el hexano fueron adquiridos de Sigma (USA).

\section{Métodos de análisis}

Determinación de la composición química: Se determinó humedad, proteínas, grasas, fibra total, cenizas y el extracto libre de nitrógeno se determinó por diferencia. Se utilizó los métodos estándar que recomienda la AOAC, $2005^{11}$.

Determinación del contenido de calcio, hierro y zinc: Se utilizó el método de la Unión Europea Norma UNE-EN 14082, determinación de elementos traza utilizando espectroscopia de absorción atómica, tras la obtención de cenizas secas.

Determinación de compuestos fenólicos mediante espectroscopia: Después de la extracción por ultrasonido, los extractos acuosos fueron centrifugados y se recogió el sobrenadante. El contenido de compuestos fenólicos totales se determinó por el método de Folin-Ciocalteu $^{12}$. Se trató $40 \mu \mathrm{L}$ de extracto con $0,5 \mathrm{~mL}$ del reactivo de Folin-Ciocalteu y 2 $\mathrm{mL}$ de carbonato sódico al $20 \%(\mathrm{p} / \mathrm{v})$ y se llevó a $10 \mathrm{~mL}$. Transcurrida media hora se efectuó la lectura de absorbancia a $760 \mathrm{~nm}$, se utilizó como estándar al ácido gálico (GAL) y los resultados se expresaron como miligramos de ácido gálico (mg GAL) /g materia prima seca.

\section{Metodología experimental}

\section{Obtención de los extractos por extracción separación asistida por ultrasonido}

Se trabajó con $1,0 \mathrm{~g}$ de cáscara de sanky seca y molida, luego se adicionó $50 \mathrm{~mL}$ del solvente y se procedió a los diferentes tratamientos de extracción en un generador ultrasónico. El equipo es el modelo NO VC 505 de marca Sonics \& Materials, INC., U.S.A. que trabajó a $20 \mathrm{KHz}$ y $130 \mathrm{~W}$, con tiempos de sonicación (sonda en contacto con el extracto acuoso) de 45 segundos con intervalos de descanso de 60 segundos. Esto se repitió y fue constante en todos los tratamientos.

\section{Diseño experimental y análisis estadístico}

Extracción asistida por ultrasonido de los componentes fenólicos

Durante la extracción se evaluó la influencia de las variables independientes: concentración de etanol $(40,50$ y $60 \% \mathrm{v} / \mathrm{v})$, tiempo $(20,30$ y $40 \mathrm{~min})$ y temperatura $\left(25,35\right.$ y $\left.45^{\circ} \mathrm{C}\right)$. Cada 
variable independiente (factor) fue evaluada a tres niveles. Se utilizó el diseño Box Behnken (DBB), el cual reportó un total de 15 tratamientos, las corridas fueron evaluadas de forma independiente para cada extracción, tal como se describe en la tabla 1. La respuesta medida fue la cantidad de componentes fenólicos totales ( $\mathrm{mgGAL} / \mathrm{g}$ de muestra). Todas las corridas se realizaron por triplicado y en forma aleatoria.

Tabla 1. Tratamientos para la extracción de los compuestos fenólicos por ultrasonido de la cáscara de sanky seca.

\begin{tabular}{cccc}
\hline Tratamientos & $\begin{array}{c}\text { Concentración de } \\
\text { etanol }(\% \mathrm{v} / \mathrm{v})\end{array}$ & $\begin{array}{c}\text { Tiempo de } \\
\text { extracción } \\
(\mathrm{min})\end{array}$ & $\begin{array}{c}\text { Temperatura de } \\
\text { extracción }\left({ }^{\circ} \mathrm{C}\right)\end{array}$ \\
\hline M1 & 40 & 20 & 35 \\
M2 & 40 & 40 & 35 \\
M3 & 60 & 40 & 35 \\
M4 & 60 & 20 & 35 \\
M5 & 40 & 30 & 25 \\
M6 & 40 & 30 & 45 \\
M7 & 60 & 30 & 25 \\
M8 & 60 & 30 & 45 \\
M9 & 50 & 20 & 25 \\
M10 & 50 & 20 & 45 \\
M11 & 50 & 40 & 25 \\
M12 & 50 & 40 & 45 \\
M13 & 50 & 30 & 35 \\
M14 & 40 & 30 & 35 \\
M15 & 60 & 30 & 35 \\
\hline
\end{tabular}

\section{Análisis estadístico}

Los datos experimentales fueron evaluados usando un análisis de variancia (ANOVA) y la prueba de Tukey, en ambos casos se consideró un nivel de significancia de $\mathrm{p}<0.05$. Los análisis se realizaron con el software STATGRAPHICS CENTURION XV. Todos los análisis se realizaron por triplicado. 


\section{RESULTADOS Y DISCUSIÓN}

\section{Caracterización de la cáscara de sanky}

La cáscara de sanky seca y molida reportó una humedad promedio de $10,74 \%$, es un valor bajo comparado con cáscaras de otras frutas como mandarina $80,14 \%$ y naranja agria $78,60 \%{ }^{15}$. Esto indica que los solutos de la cáscara se encuentran concentrados, por tanto, la concentración de los polifenoles de la cáscara también es alta comparada con otros residuos. En la tabla 2, se aprecia la composición proximal de la cáscara de sanky seca. El extracto libre de nitrógeno fue $46,25 \%$, esto implica la presencia de hidrocoloides y/o compuestos solubles en agua, tal como lo reporta Lipe ${ }^{6}$ al encontrar 38,24\% de un hidrocoloide en la pulpa del sanky. También podría poseer hemicelulosa. Asimismo, se hallaron 16,39 \% y 14,75\% de fibra y cenizas en la cáscara, respectivamente. La fibra o fracción insoluble es casi la tercera parte de la fracción soluble, así que ambas partes (representan casi el 62,64 \%) aportarían a mejorar el tránsito intestinal y como protector de la mucosa gástrica, por ende, serían de gran aporte para mejorar el proceso digestivo.

Tabla 2. Composición química de la cáscara de sanky seca (gramos/100gmuestra).

\begin{tabular}{cc}
\hline Determinación & Porcentaje $\left(^{*}\right)$ \\
\hline Humedad & 10,74 \\
Proteínas & 9,19 \\
Cenizas & 14,75 \\
Grasa & 2,68 \\
Fibra & 16,39 \\
E.L.N. & 46,25 \\
\hline
\end{tabular}

(*) Son valores promedios de tres repeticiones

Respecto al contenido de cenizas podría incluirse la presencia de calcio y potasio, que según Nolazco y Guevara ${ }^{3}$ encontraron 249 ppm de calcio y 417 ppm de potasio en el néctar de sanky. Por otra parte, en las semillas de sanky, también se encontraron minerales como calcio, hierro y zinc, en concentraciones de 207,81; 39,36 y 9,4 ppm, respectivamente, como se muestra en la tabla 3. Esto permite deducir que también en la cáscara del fruto se encuentran altas concentraciones de estos micronutrientes, especialmente calcio que podría ser utilizado en la elaboración de otros productos.

Tabla 2. Composición química de la cáscara de sanky seca (gramos/100gmuestra).

\begin{tabular}{cc}
\hline Metales & Contenido en ppm $\left(^{*}\right)$ \\
\hline Calcio & 207,81 \\
Hierro & 39,36 \\
Zinc & 9,4 \\
\hline
\end{tabular}

(*) Se reporta el promedio de dos repeticiones 
En general, las frutas y sus derivados no son fuentes de proteínas, ni grasas, es así que la cáscara contiene $9,19 \%$ de proteínas y $2,68 \%$ de grasas, según se reportó en la tabla 2 .

Estos resultados muestran que la cáscara de sanky seca y molida presenta componentes que ayudarían a mejorar el tránsito intestinal y a proteger la mucosa gástrica y por su contenido de cenizas es una buena fuente de minerales como calcio, hierro y zinc. Se podría utilizar como ingrediente para elaborar alimentos funcionales $y / 0$ otros productos en la industria cosmética y farmacéutica.

\section{Extracción por ultrasonido de los componentes fenólicos de la cáscara de sanky}

En la mayoría de los tratamientos, los polifenoles totales presentaron mayores valores que los reportados por Alanoca ${ }^{13}$ quien utilizó el método tradicional de maceración con etanol para la extracción de los compuestos fenólicos.

Según los datos de la figura 1, el extracto M11, con $50 \%$ v/v de etanol, 40 minutos de tiempo de extracción y $25^{\circ} \mathrm{C}$, fue la que presentó el mayor rendimiento de extracción reflejada en la mayor cantidad de polifenoles totales, reportó 43,9 mg GAL/g de cáscara seca, mostrando un amplio margen muy superior al resto de tratamientos. Los extractos M3 (60\% etanol, 40 minutos y $\left.35^{\circ} \mathrm{C}\right)$, M6 $\left(40 \%\right.$ etanol, 30 minutos y $\left.45^{\circ} \mathrm{C}\right)$ y M9 $(50 \%$ etanol, 20 minutos y $25^{\circ} \mathrm{C}$ ) mostraron valores muy similares, 31,5; 32,9 y 32,6 mg GAL/g de materia seca, respectivamente. Entre estos tres tratamientos no hubo diferencias significativas en el contenido de fenoles totales, por tanto, también presentarían actividad antioxidante muy similar. De este grupo, el extracto M9, sería el más recomendable para extraer los polifenoles a un menor tiempo (20 minutos) y menor temperatura $\left(25^{\circ} \mathrm{C}\right)$, la temperatura es un factor que afecta en forma desfavorable la estabilidad de los fenoles. El extracto M4 (60\% de etanol, 20 minutos y $35^{\circ} \mathrm{C}$ ) reportó el menor contenido de polifenoles, 14,2 mg GAL/g de materia seca, esto se puede deber a la mayor concentración del etanol, ya que la solubilidad de los fenólicos tiende a disminuir, además el tiempo de extracción fue de apenas 20 minutos. La solubilidad de estas sustancias disminuye en solventes menos polares como metanol y etanol, se recomienda utilizar mezclas con agua, para incrementar la polaridad como acetona:agua y/o etanol:agua para mejorar la extracción de polifenoles ${ }^{5}$. 


\section{Contenido de compuestos fenólicos totales}

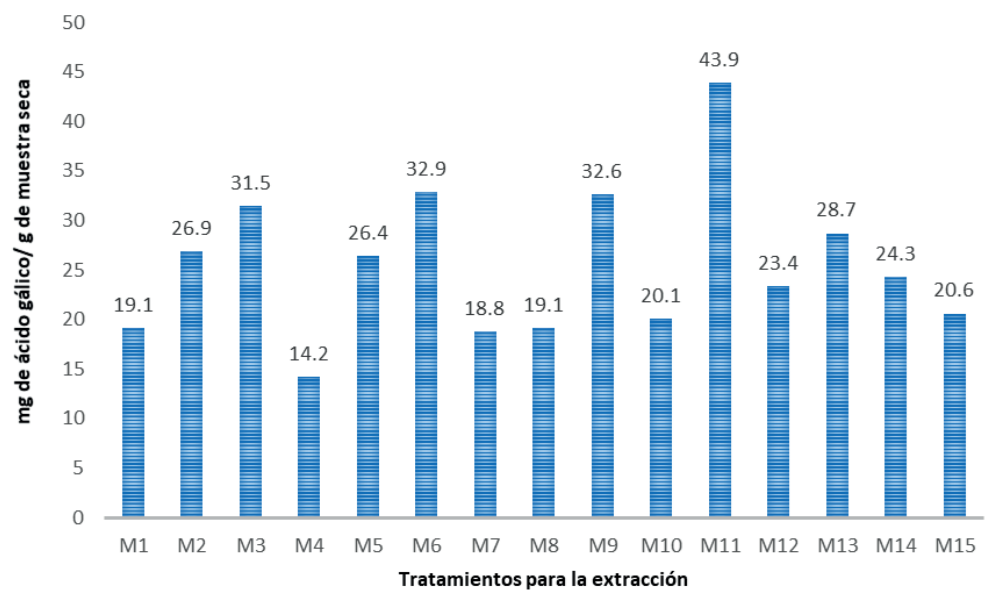

Figura 1. Contenido de compuestos fenólicos totales en las cáscaras de sanky seca y molida en los diferentes tratamientos.

Dado que los extractos M11 (50\% etanol, 40 minutos y $\left.25^{\circ} \mathrm{C}\right)$ y M9 (50\% etanol, 20 minutos y $25^{\circ} \mathrm{C}$ ) fueron los de mayor contenido de fenoles totales, se deduce que el tiempo de extracción es la variable independiente de mayor influencia seguido de la concentración del solvente. En las cáscaras de varios frutos, se reporta presencia de sustancias bioactivas en mayor concentración que en la misma pulpa, en el caso de las cáscaras de plátano, sandía y uva se reportaron valores de polifenoles como ácido gálico equivalente (AGE) de 24,42; 26,73 y $113,40 \mathrm{mg} \mathrm{AGE} / \mathrm{g}$ de muestra, respectivamente ${ }^{7}$ así como la cáscara de mandarina común y de toronja que según Ordóñez et al. ${ }^{9}$, presentaron 3,22 y 3,08 mg AGE/ g muestra, respectivamente. En todos los casos, los extractos de cáscara de sanky presentaron mayor contenido de polifenoles que las cáscaras de frutas antes mencionadas. En las cáscaras de frutas cítricas el contenido es mucho menor aún. Esto podría deberse a que la planta de sanky, por ser una cactácea requiere de poca agua para su adaptación y crecimiento, es así que los solutos se encuentran altamente concentrados en comparación con otros residuos frutales. El contenido de fenoles en el sanky puede variar debido a las características del suelo, a la época de siembra y/o condiciones de post cosecha. En el extracto de cáscara de granada se reportó $165,4 \mathrm{mg}$ de GAL/g de extracto en peso seco $^{14}$, valor bastante superior al encontrado en la cáscara de sanky. Sin embargo, estos polifenoles en las plantas pueden estar en forma simple, polimerizados o incluso pueden estar ligados a proteínas o carbohidratos, lo cual implica que lograr un sistema de extracción para estructuras tan distintas resulta ser muy complejo.

Al respecto Balasundram et $a l^{8}$ mencionan que los compuestos fenólicos extraídos de extractos vegetales tienen correlación positiva con la actividad antioxidante y podrían actuar como agentes que previenen la oxidación a nivel celular. Por tanto, todos los extractos obtenidos por ultrasonido también tendrían actividad antioxidante. 


\section{CONCLUSIONES}

La extracción asistida por ultrasonido (EAU) para recuperar los componentes fenólicos de la cáscara de sanky, utilizando el diseño de Box Behnken, reportó la mejor eficiencia utilizando $50,0 \% \mathrm{v} / \mathrm{v}$ de etanol, con un tiempo de extracción de 40 minutos y una temperatura de $25{ }^{\circ} \mathrm{C}$, mostrando una diferencia significativa, muy superior al resto de tratamientos. En esta metodología, el tiempo de extracción fue el factor más importante, seguido de la concentración del solvente (etanol).

\section{AGRADECIMIENTO}

Al Departamento Académico de Química, Facultad de Ciencias de la Universidad Nacional Agraria La Molina por su apoyo con el equipo de extracción por ultrasonido.

\section{REFERENCIAS BIBLIOGRÁFICAS}

1. Chen W, Wang W, Zhang H. Optimization of ultrasonic-assisted extraction of watersoluble polysaccharides from Boletus edulis mycelia using response surface methodology. Carbohydr Polym. 2012; 87: 614-619.

2. Medina-Torres N, Ayora-Talavera T, Espinosa-Andrews H. Ultrasound Assisted Extraction for the Recovery of Phenolic Compounds from Vegetable Sources. Agronomy. 2017;7(3): 47

3. Nolazco D, Guevara A. Estudio de las principales características fisicoquímicas y comportamiento del néctar de sanky (Corryocactus brevistylus subsp. puquiensis (Rauh \& Backeberg) Ostolaza) en almacenamiento. Anales Científicos UNALM. 2009; 70 (4):1-11.

4. Matos R, Paredes J, Gonzales L. Determinación de la Capacidad Antioxidante de los Compuestos Fenólicos del Sancayo (Corryocactus brevistylus). Revista de Investigación en Ciencia y Tecnología de Alimentos. 2010;1(1):66-71

5. Zou T, Xia E, He T, Huang M, Jia Q, Li H. Ultrasound-assisted extraction of Mangiferin from Mango (Mangifera indica L.) leaves using response surface methodology. Molecules. 2014;19(2):1411-21.

6. Lipe C. Efecto hepatoprotector del zumo de fruto de Corryocactus brevistylus (sanky) en ratones con daño hepático inducido por etanol. [Tesis para licenciada en Nutrición]. Lima: Universidad Nacional Mayor de San Marcos; 2016.

7. Hurtado R, Ortiz L. Componentes Fitoquímicos y Capacidad Antioxidante de cáscaras de frutas de mayor consumo en el Perú. [Tesis para licenciada en Nutrición y Dietética]. Lima, Perú: Universidad Peruana de Ciencias Aplicadas; 2018.

8. Balasundram N, Sundram K, Samman S. Phenolic compounds in plants and agriindustrial by-products: Antioxidant activity, occurrence and potencial uses. Food Chem. 2006; 99: 191-203 
9. Ordóñez-Gómez E, Reátegui-Díaz D, Villanueva-Tiburcio J. Total polyphenols and antioxidant capacity of peel and leaves in twelve citrus. Scientia Agropecuaria. 2018; 9(1):113 - 121 .

10. International Solid Waste Association. Waste Atlas 2013 Report. [Internet]. International Solid Waste Association [citado 7 feb 2019]. Disponible en: https://www.iswa.org/ fileadmin/galleries/News/WASTE_ATLAS_2013_REPORT.pdf [Accessed 7 February 2019].

11. AOAC. Official Methods of Analysis. 18th. Edition. Gaithersburg, Maryland, USA: Association of Official Analytical Chemists; 2005.

12. Waterhouse A. Determination of total phenolics. Curr Prot Food Anal Chem. 2002; 6(1):I1.1.1-I1.1.8

13. Alanoca S. Evaluación del contenido de ácido ascórbico y polifenoles totales del sancayo (Corryocactus brevistylus) y determinación de su actividad antioxidante y genotoxicidad en linfocitos de sangre periférica [Tesis Ingeniera en Biotecnología]. Arequipa, Perú: Universidad Católica de Santa María; 2014.

14. Rodríguez J, López M. Extracción por ultrasonido de los polifenoles de la fruta Punica granatum (granada). Rev Cubana de Farm. 2014; 48(2):469-476.

15. Escobar Blanco M. Extracción de compuestos fenólicos de las cáscaras de cítricos producidos en México. [Tesis para optar el Titulo de Maestra en Ciencia de Alimentos]. México DF, México: Escuela de posgrado del Instituto Politécnico Nacional; 2010. 